\title{
INEQUALITIES AND MONOTONICITY RESULTS FOR ZEROS OF MODIFIED BESSEL FUNCTIONS OF PURELY IMAGINARY ORDER*
}

BY

\author{
ANDREA LAFORGIA
}

University of Torino, Torino, Italy

Summary. Let $x_{k}(\nu)$ and $x_{k}^{\prime}(\nu)$ denote the $k$ th positive zeros, in decreasing order, of the modified Bessel function $K_{i \nu}(x)$ of purely imaginary order and of its derivative $K_{i \nu}^{\prime}(x)=\frac{d}{d x} K_{i \nu}(x)$, respectively. We show that for $k=1,2, \ldots$ and $0<\nu<\infty, x_{k}(\nu)<$ $x_{k}^{\prime}(\nu)<\nu$ and $x_{k}(\nu) / x_{k+1}(\nu)$ and $x_{k}(\nu)-x_{k+1}(\nu)$ decrease as $k$ increases. Some related results are mentioned for the zeros of $K_{i \nu}^{\prime}(x)$ and the chain of inequalities $\left|K_{i \nu}\left(x_{1}^{\prime}(\nu)\right)\right|>$ $\left|K_{i \nu}\left(x_{2}^{\prime}(\nu)\right)\right|>\cdots>\left|K_{i \nu}\left(x_{n}^{\prime}(\nu)\right)\right|>\left|K_{i \nu}\left(x_{n+1}^{\prime}(\nu)\right)\right|>\cdots$ is established.

1. Introduction. We are concerned in this paper with some inequalities and monotonicity results for the zeros of the modified Bessel function $K_{\mu}(x)$. It is known [5, p. 88] that for real $x$ the function $K_{\mu}(x)$ has no zeros if $\mu$ is not purely imaginary. However when $\mu$ is a pure imaginary number $(\mu=i \nu)$ the function $K_{i \nu}(x)$ vanishes at $+\infty$ and has infinitely many positive zeros whose only point of accumulation is $x=0$.

These zeros occur in certain physical problems such as in the determination of the bound states for an inverse square potential with hard core in the Schrödinger equation [4]. See [5] for other possible useful applications.

We indicate with $x_{k}(\nu)(k=1,2, \cdots)$ the positive zeros of $K_{i \nu}(x)$ in decreasing order $\left(x_{1}(\nu)>x_{2}(\nu)>\cdots>x_{n}(\nu)>x_{n+1}(\nu)>\cdots>0\right)$ and deal with some inequalities and monotonicity results for $x_{k}(\nu)$. The behaviour of $x_{k}(\nu)$ has been investigated by Ferreira and Sesma [4] and later by Lewis and Muldoon [8]. Here we consider the sequence $\left\{x_{k}^{\prime}(\nu)\right\}_{k=1}^{\infty}$ of the positive zeros of $K_{i \nu}^{\prime}(x)=\frac{d}{d x} K_{i \nu}(x)$ ordered in the following way

$$
x_{1}^{\prime}(\nu)>x_{2}^{\prime}(\nu)>\cdots>x_{n}^{\prime}(\nu)>x_{n+1}^{\prime}(\nu)>\cdots>0
$$

and show, among other things, that the first turning value $K_{i \nu}\left(x_{1}^{\prime}(\nu)\right)$ is a maximum and that the inequalities

$$
x_{k}(\nu)<x_{k}^{\prime}(\nu)<\nu, \quad 0<\nu<\infty, k=1,2, \cdots
$$

\footnotetext{
*Received February 22, 1985.
} 
hold. Clearly this result is more illuminating than the inequality $x_{1}(\nu)<\nu$ established in [4]. We study also the connection between the zeros $x_{k}(\nu)$ of $K_{i \nu}(x)$ and $x_{k}^{\prime}(\nu)$ of $K_{i \nu}^{\prime}(x)$ showing that between two consecutive zeros of $K_{i \nu}(x)$ there lies one and only one zero of $K_{i \nu}^{\prime}(x)$.

We are interested also in some monotonicity results for the ratio $x_{k}(\nu) / x_{k+1}(\nu)$ and the difference $x_{k}(\nu)-x_{k+1}(\nu)$. The method employed for these results is that which was used by Lorch [9] for the zeros of Bessel functions and by Elbert and Laforgia [3] for the zeros of ultraspherical polynomials.

Finally in section 4 we prove the chain of inequalities

$$
\left|K_{i \nu}\left(x_{1}^{\prime}(\nu)\right)\right|>\left|K_{i \nu}\left(x_{2}^{\prime}(\nu)\right)\right|>\cdots>\left|K_{i \nu}\left(x_{n}^{\prime}(\nu)\right)\right|>\left|K_{i \nu}\left(x_{n+1}^{\prime}(\nu)\right)\right|>\cdots
$$

and mention some possible extensions to $x_{k}^{\prime}(\nu)$ of the monotonicity results obtained for $x_{k}(\nu)$.

The principal tools used here are the differential equation [12, p. 77]

$$
x^{2} y^{\prime \prime}+x y^{\prime}-\left(x^{2}-v^{2}\right) y=0
$$

satisfied by $K_{i \nu}(x)$, the Sturm comparison theorem in a form given by Szegö and the Sonine-Polya theorem. These theorems will be enunciated in Sections 3 and 4, respectively.

2. The inequalities. A result established in [4] asserts that the first positive zero $x_{1}(\nu)$ satisfies the inequality

$$
x_{1}(\nu)<\nu, \quad 0<\nu<\infty .
$$

Since the sequence of zeros $x_{k}(\nu)$ of $K_{i \nu}(x)$ is infinite and $x=0$ is the only point of accumulation, then by Rolle's theorem applied to each interval $\left(x_{k}(\nu), x_{k-1}(\nu)\right)$ we get that also the sequence of the zeros of $K_{i \nu}^{\prime}(x)$ is infinite and $x=0$ is a point of accumulation for this sequence.

We now give further information on the behaviour of $K_{i v}(x)$ for large values of $x$ and we prove a more precise result than (2.1).

From the relation $[5$, p. 56]

$$
\lim _{x \rightarrow+\infty} \frac{K_{i \nu}(x)}{\sqrt{\pi / 2 x} e^{-x}}=1
$$

we obtain immediately that $K_{i \nu}(x)$ tends to zero when $x$ tends to infinity and that it is ultimately positive. This proves that on the right-hand side of $x_{1}(\nu)$ there is at least one zero, say $a$, of $K_{i \nu}^{\prime}(x)$ which is a maximum point for $K_{i \nu}(x)$. Moreover $K_{i \nu}(a)$ is positive, $K_{i \nu}^{\prime}(a)=0$ and $K_{i \nu}^{\prime \prime}(a)$ is negative. From the differential equation (1.1) satisfied by $K_{i \nu}(x)$ it follows that

$$
a^{2} K_{i \nu}^{\prime \prime}(a)=\left(a^{2}-\nu^{2}\right) K_{i \nu}(a),
$$

so that $a^{2}-\nu^{2}<0$ and hence $a<\nu$.

Thus the value $\nu$ is an upper bound for every maximum point of $K_{i \nu}(x)$. Moreover since $\lim _{x \rightarrow \infty} K_{i \nu}(x)=0^{+}$, hence on the right-hand side of every minimum point of $K_{i \nu}(x)$, say $b>x_{1}(\nu)$, there exists a maximum point. Therefore we can conclude that the value $\nu$ is an upper bound for every zero of $K_{i \nu}^{\prime}(x)$ and that there exist a finite number of zeros of $K_{i \nu}^{\prime}(x)$ which are greater than $x_{1}(\nu)$. 
This shows that, like $K_{i v}(x)$, the function $K_{i \nu}^{\prime}(x)$ has infinitely many positive zeros whose only point of accumulation is $x=0$. Hence we can indicate by $\left\{x_{k}^{\prime}(\nu)\right\}_{k=1}^{\infty}$ the infinite sequence of such zeros in decreasing order, that is

$$
x_{1}^{\prime}(\nu)>x_{2}^{\prime}(\nu)>\cdots>x_{n}^{\prime}(\nu)>x_{n+1}^{\prime}(\nu)>\cdots
$$

and conclude that $x_{1}^{\prime}(\nu)$ is a maximum point for $K_{i \nu}(x)$. Thus we have established the following result.

TheOREM 2.1. Let $x_{1}(\nu)$ and $x_{1}^{\prime}(\nu)$ be the largest positive zeros of $K_{i \nu}(x)$ and $K_{i \nu}^{\prime}(x)$, respectively, $0<\nu<\infty$. Then

$$
x_{1}(\nu)<x_{1}^{\prime}(\nu)<\nu, \quad 0<\nu<\infty .
$$

Remark 2.1. A simple application of Rolle's theorem on the interval $\left(x_{k+1}(\nu), x_{k}(\nu)\right)$ shows that the inequality $x_{1}(\nu)<x_{1}^{\prime}(\nu)$ can be extended to all $x_{k}(\nu)$ and $x_{k}^{\prime}(\nu)$, so that more generally we get

$$
x_{k}(\nu)<x_{k}^{\prime}(\nu)<\nu, \quad k=1,2, \ldots, \quad 0<\nu<\infty .
$$

THEOREM 2.2. Between two consecutive zeros of $K_{i \nu}(x)$ there lies one and only one zero of $K_{i \nu}^{\prime}(x)$.

Proof. By Rolle's theorem there exists one such zero of $K_{i \nu}^{\prime}(x)$. If there exists more than one, then there must be at least three. But for at least one of these three zeros of $K_{i \nu}^{\prime}(x)$, say $b, K_{i \nu}^{\prime \prime}(b)$ and $K_{i \nu}(b)$ would be of the same sign. On the other hand (2.2) with $a$ replaced by $b$ shows that this contradicts the property $b<\nu$ proved in Theorem 2.1. The proof of Theorem 2.2 is complete.

Corollary 2.1. The zeros of $K_{i \nu}(x)$ and $K_{i \nu}^{\prime}(x)$ separate each other.

Proof. This is an immediate consequence of Theorem 2.2 and the fact that by this theorem there exists exactly one zero $x_{1}^{\prime}(\nu)$ of $K_{i \nu}^{\prime}(x)$ on the interval $\left(x_{1}(\nu), \infty\right)$.

3. Monotonicity results. We are interested in this section in the monotonicity in $k$ of the ratio $x_{k}(\nu) / x_{k+1}(\nu)$ and of the difference $x_{k}(\nu)-x_{k+1}(\nu)(k=1,2, \ldots)$ between two consecutive zeros of $K_{i \nu}(x)$. The tool used here is the Sturm comparison theorem that for our purposes it is useful to enunciate in Szegö's form, [10, p. 19].

LEMma 3.1. (Sturm comparison theorem). Let the functions $y(x)$ and $Y(x)$ be nontrivial solutions of the differential equations

$$
y^{\prime \prime}(x)+f(x) y=0 ; \quad Y^{\prime \prime}(x)+F(x) Y(x)=0
$$

and let them have consecutive zeros at $x_{1}, x_{2}, \ldots, x_{m}$ and $X_{1}, X_{2}, \ldots, X_{m}$ respectively on an interval $(a, b)$. Suppose that $f(x)$ and $F(x)$ are continuous, that $f(x)<F(x)$, $a<x<x_{m}$, and that

$$
\lim _{x \rightarrow a^{+}}\left[y^{\prime}(x) Y(x)-y(x) Y^{\prime}(x)\right]=0 .
$$

Then

$$
X_{k}<x_{k}, \quad k=1,2, \ldots, m .
$$


Theorem 3.1. Let $W_{i \nu}(x)$ and $Z_{i \nu}(x)$ be modified Bessel functions of purely imaginary order, i.e., any two solutions of differential equation (1.1), not necessarily linearly independent. Moreover let $a_{k}(\nu)$ and $b_{k}(\nu)$ be their respective positive zeros, with $b_{k}(\nu+\varepsilon)<a_{m}(\nu)$ for a fixed pair of positive integers $k$ and $m$ and some $\varepsilon \geqslant 0$. Then

$$
\frac{b_{k}(\nu+\varepsilon)}{b_{k+1}(\nu+\varepsilon)}<\frac{a_{m}(\nu)}{a_{m+1}(\nu)} \text {. }
$$

Proof. The functions $W_{i \nu}\left(e^{x}\right)$ and $Z_{i \nu}\left(e^{x}\right)$ satisfy the differential equation

$$
y^{\prime \prime}+\phi_{\nu}(x) y=0
$$

where $\phi_{\nu}(x)=-e^{2 x}+\nu^{2}$.

Besides (3.1) we consider the differential equation

$$
z^{\prime \prime}+\phi_{\nu+\varepsilon}(x-h) z=0
$$

satisfied by $Z_{i(\nu+\varepsilon)}\left(e^{x-h}\right)$ where

$$
h=\log a_{m}(\nu)-\log b_{k}(\nu+\varepsilon)>0 .
$$

Clearly $\phi_{\nu}(x)<\phi_{\nu+\varepsilon}(x-h)$ for $\varepsilon \geqslant 0$, so we can compare the differential equations (3.1) and (3.2). The functions $W_{i \nu}\left(e^{x}\right)$ and $Z_{i(\nu+\varepsilon)}\left(e^{x-h}\right)$ both vanish at $\log a_{m}(\nu)=\log b_{k}(\nu+$ $\varepsilon)+h$ and by the Sturm comparison theorem (Lemma 3.1) the next zero of $Z_{i(\nu+\varepsilon)}\left(e^{x-h}\right)$ precedes the next zero of $W_{i \nu}\left(e^{x}\right)$. Hence $\log b_{k-1}(\nu+\varepsilon)+h<\log a_{m-1}(\nu)$, leading to

$$
\frac{b_{k-1}(\nu+\varepsilon)}{b_{k}(\nu+\varepsilon)}<\frac{a_{m-1}(\nu)}{a_{m}(\nu)}, \quad \nu>0, \quad b_{k}(\nu+\varepsilon)<a_{m}(\nu), \quad \varepsilon \geqslant 0 .
$$

The proof of Theorem 3.1 is complete.

COROLlaRY 3.1. Under the hypotheses of Theorem 3.1

$$
\frac{x_{k-1}(\nu)}{x_{k}(\nu)}<\frac{x_{k-2}(\nu)}{x_{k-1}(\nu)}, \quad \nu>0 .
$$

Proof. The result follows from Theorem 3.1 in the particular case $W_{i \nu}(x) \equiv Z_{i \nu}(x) \equiv$ $K_{i \nu}(x)$, with $\varepsilon=0, m=k-1$ and $b_{k}(\nu) \equiv a_{k}(\nu) \equiv x_{k}(\nu)$.

Remark 3.1. We observe that the main idea used in the proof of Theorem 3.1 has been used already by Szegö and Turán [11] for the ultraspherical polynomials and subsequently extended to the zeros of other special functions, (see [2, 3, 7, 9]). L. Lorch called this method "the use of the simultaneous monotonicity in $\nu$ and $x$ of the function $\psi_{\nu}(x)$ in the differential equation $y^{\prime \prime}+\psi_{\nu}(x) y=0$."

THEOREM 3.2. Under the conditions of Theorem 3.1 we get

$$
b_{k-1}(\nu+\varepsilon)-b_{k}(\nu+\varepsilon)<a_{m-1}(\nu)-a_{m}(\nu) .
$$

Proof. The proof runs along the same lines as that of Theorem 3.1.

We consider the differential equation

$$
y^{\prime \prime}+\gamma_{\nu}(x) y=0
$$

where $\gamma_{\nu}(x)=-1+\frac{1 / 4+\nu^{2}}{2^{2}}$, satisfied by $x^{1 / 2} K_{i \nu}(x)$ and in addition the new differential equation

$$
z^{\prime \prime}+\gamma_{\nu+\varepsilon}(x-h) z=0
$$


where

$$
h=a_{m}(\nu)-b_{k}(\nu+\varepsilon)>0,
$$

satisfied by $Z_{i(\nu+\varepsilon)}(x-h)$. We get immediately $\gamma_{\nu}(x)<\gamma_{\nu+\varepsilon}(x-h)$. Moreover $W_{i \nu}(x)$ and $Z_{i(\nu+\varepsilon)}(x-h)$ both vanish at $a_{m}(\nu)$, so an application of the Sturm comparison theorem gives

$$
b_{k-1}(\nu+\varepsilon)-b_{k}(\nu+\varepsilon)<a_{m-1}(\nu)-a_{m}(\nu), \quad \nu>0, \quad b_{k}(\nu+\varepsilon)<a_{m}(\nu),
$$$$
\varepsilon \geqslant 0 \text {, }
$$

which is the desired result.

Corollary 3.2. Under the hypotheses of Theorem 3.2,

$$
x_{k-1}(\nu)-x_{k}(\nu)<x_{k-2}(\nu)-x_{k-1}(\nu) .
$$

Proof. The result follows by applying Theorem 3.2 with

$$
W_{i \nu}(x) \equiv Z_{i \nu}(x) \equiv K_{i \nu}(x) \text { and } \varepsilon=0, \quad m=k-1, \quad a_{k}(\nu) \equiv b_{k}(\nu) \equiv x_{k}(\nu) \text {. }
$$

4. Further results. Further information on the behaviour of the function $K_{i \nu}(x)$ with respect to $x$ can be drawn from a simple application of the following classical result $[10, \mathrm{p}$. 166].

LEMMA 4.1. (Sonine-Polya theorem). Let $y=y(x)$ satisfy the differential equation

$$
\frac{d}{d x}\left[p(x) \frac{d y}{d x}\right]+P(x) y=0
$$

where $p(x)$ and $P(x)$ are positive in $(a, b)$.

Suppose that the function $Q(x)=p(x) P(x)$ has a continuous derivative of a constant sign in $(a, b)$. Then the successive relative maxima of $|y(x)|$, as $x$ increases from $a$ to $b$, form an increasing or decreasing sequence according as $Q(x)$ decreases or increases.

We write the differential equation (1.2) in the following self-adjoint form

$$
\frac{d}{d x}\left(x \frac{d y}{d x}\right)+\left(-x+\frac{\nu^{2}}{x}\right) y=0, \quad y=K_{i \nu}(x)
$$

and observe that with the notations of Lemma 4.1, $Q(x)=p(x) P(x)=-x^{2}+\nu^{2}$ which clearly decreases as $x$ increases $(x>0)$. Therefore an application of Lemma 4.1 gives the following chain of inequalities

$$
\left|K_{i \nu}\left(x_{1}^{\prime}(\nu)\right)\right|>\left|K_{i \nu}\left(x_{2}^{\prime}(\nu)\right)\right|>\cdots>\left|K_{i \nu}\left(x_{n}^{\prime}(\nu)\right)\right|>\left|K_{i \nu}\left(x_{n+1}^{\prime}(\nu)\right)\right|>\cdots .
$$

Another result that we can prove for the zeros $x_{k}^{\prime}(\nu)$ of $K_{i \nu}^{\prime}(x)$ is the following.

THEOREM 4.1. Let $a_{k}^{\prime}(\nu)$ and $b_{k}^{\prime}(\nu)$ be the $k$ th positive zeros of the derivatives with respect to $x$ of the general modified Bessel functions $W_{i \nu}(x)$ and $Z_{i \nu}(x)$, respectively, and let, for a fixed pair of positive integers $k$ and $m$,

$$
b_{k}^{\prime}(\nu+\varepsilon)<a_{m}^{\prime}(\nu), \quad \varepsilon \geqslant 0 .
$$


Then

$$
\frac{b_{k}^{\prime}(\nu+\varepsilon)}{b_{k+1}^{\prime}(\nu+\varepsilon)}<\frac{a_{m}^{\prime}(\nu)}{a_{m+1}^{\prime}(\nu)} .
$$

We only indicate the proof of Theorem 4.1, because it is analogous to that of Theorem 3.1. The only difference in the proofs is that instead of Lemma 3.1 we must use a more general result (Sturm-Picone theorem) [6, pp. 225-226] which enables us to compare, reaching the same conclusions as in Lemma 3.1, the differential equation (4.1) and a new equation $(d / d x)(q(x) d v / d x)+Q(x) v=0$, when in $(a, b), p(x)>q(x)>0$ and $0<P(x)<$ $Q(x)$.

From the differential equation (3.1) we find immediately that $z(x)=(d / d x) K_{i \nu}\left(e^{x}\right)$ satisfies

$$
\frac{d}{d x}\left[\frac{d z / d x}{\nu^{2}-e^{2 x}}\right]+z=0 .
$$

Thus, following the lines of the argument given in the proof of Theorem 3.1, we have the conclusion of Theorem 4.1 .

Clearly new results can be found using other differential equations. For example from (3.2) satisfied by $y=x^{1 / 2} K_{i \nu}(x)$ we can obtain similar properties to that of Theorem 3.2, for the zeros of the function $K_{i \nu}(x)+2 x K_{i \nu}^{\prime}(x)$.

\section{REFERENCES}

[1] M. Abramowitz and I. A. Stegun, eds., Handbook of mathematical functions, Applied Mathematics Series 55, National Bureau of Standards, Washington, 1964

[2] S. Ahmed, A. Laforgia and M. E. Muldoon, On the spacing of the zeros of some classical orthogonal polynomials, J. London Math. Soc. (2) 25, 246-252 (1982)

[3] A. Elbert and A. Laforgia, Some monotonicity properties of the zeros of ultraspherical polynomials, Acta Math. Hungar. (to appear)

[4] E. M. Ferreira and J. Sesma, Zeros of modified Hankel functions, Numer. Math. 16, 278-284 (1970)

[5] A. Gray, G. B. Mathews and T. M. Macrobert, A Treatise on Bessel Functions and their Applications to Physics, Macmillan, London, 1952

[6] E. L. Ince, Ordinary differential equations, Longmans, London, 1927; reprinted, Dover, New York, 1944

[7] A. Laforgia, Sturm theory for certain classes of Sturm-Liouville equations and Turanians and Wronskians for the zeros of derivative of Bessel functions, Indag. Math. 44, 295-301 (1982)

[8] J. T. Lewis and M. E. Muldoon, Monotonicity and convexity properties of zeros of Bessel functions, SIAM J. Math. Anal. 8, 171-178 (1977)

[9] L. Lorch, Elementary comparison techniques for certain classes of Sturm-Liouville equations, Differential Equations, Proc. Intern. Conf., Uppsala, 1977, Sympos. Univ. Upsaliensis Ann. Quingentesimum Celebrantis 7. Almqvist and Wiksell, Stockholm, 1977, pp. 125-133

[10] G. Szegö, Orthogonal Polynomials, Amer. Math. Soc. Colloq. Publ. 23, 4th ed., Amer. Math. Soc., Providence, R. I., 1975

[11] G. Szegö and P. Turán, On the monotone convergence of certain Riemann Sums, Publ. Math. Debrecen 8, $326-335$ (1961)

[12] G. N. Watson, A Treatise on the Theory of Bessel functions, 2nd ed., Cambridge University Press, Cambridge, England, 1944 